\title{
“TORINO 1911" PROJECT: A CONTRIBUTION OF A SLAM-BASED SURVEY TO EXTENSIVE 3D HERITAGE MODELING
}

\author{
F. Chiabrando ${ }^{a^{*}}$, C. Della Coletta ${ }^{b}$, G. Sammartano ${ }^{a^{*}}$, A. Spanò ${ }^{a}$, A. Spreafico ${ }^{a}$ \\ ${ }^{\text {a }}$ DAD, Department of Architecture and Design, Politecnico di Torino \\ (filiberto.chiabrando, giulia.sammartano, antonia.spano, alessandra.spreafico)@polito.it

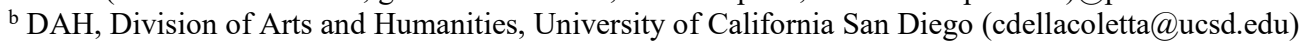

\section{Commission II, WG II/8}

KEY WORDS: SLAM, photogrammetry, LiDAR, Zeb Revo RT, Mobile Mapping Systems, landscape, Cultural Heritage

\begin{abstract}
:
In the framework of the digital documentation of complex environments the advanced Geomatics researches offers integrated solution and multi-sensor strategies for the 3D accurate reconstruction of stratified structures and articulated volumes in the heritage domain. The use of handheld devices for rapid mapping, both image- and range-based, can help the production of suitable easy-to use and easynavigable 3D model for documentation projects. These types of reality-based modelling could support, with their tailored integrated geometric and radiometric aspects, valorisation and communication projects including virtual reconstructions, interactive navigation settings, immersive reality for dissemination purposes and evoking past places and atmospheres. The aim of this research is localized within the "Torino 1911" project, led by the University of San Diego (California) in cooperation with the PoliTo. The entire project is conceived for multi-scale reconstruction of the real and no longer existing structures in the whole park space of more than $400,000 \mathrm{~m}^{2}$ for a virtual and immersive visualization of the Turin 1911 International "Fabulous Exposition" event, settled in the Valentino Park. Particularly, in the presented research, a 3D metric documentation workflow is proposed and validated in order to integrate the potentialities of LiDAR mapping by handheld SLAM-based device, the ZEB REVO Real Time instrument by GeoSLAM (2017 release), instead of TLS consolidated systems. Starting from these kind of models, the crucial aspects of the trajectories performances in the 3D reconstruction and the radiometric content from imaging approaches are considered, specifically by means of compared use of common DSLR cameras and portable sensors.
\end{abstract}

\section{INTRODUCTION}

The ability of 3D models to suggest real-world scenarios or evoking past atmospheres is an essential topic in case of immersive and interactive navigations on virtual reconstructions regarding cultural heritage scenarios. The Geomatics more and more are able to test advanced integrated methods, to provide worthy impactful but easy-handy 3D models and to support these communication systems, which could be implemented on userfriendly platforms, interactive digital displays, museum installations and websites and web-based 3D applications (Evans, Romeo, Bahrehmand, Agenjo, \& Blat, 2014; Spanò, Chiabrando, Dezzani, \& Prencipe, 2016). They are able to flank, making even more efficient, the long-established communication approaches and to offer valuable solutions in educational and dissemination purposes. Particularly for heritage ancient structures and artefacts, the demand of easy-to-use and easymanageable high-resolution models made of dense point clouds and meshed surfaces is very significant (million points/triangles/vertices with associated texture attributes) and it is usual as well that other source data or media need to be connected with the spatial reconstruction (Lee, Lavoué, \& Dupont, 2012; Potenziani et al., 2015). New scenarios will be opened by digital 3D reconstruction in the learning approaches of cultural heritage. In fact, a digital visualization with a possible interaction of the user with these structures allows analysing and annotating them, and making for example queries and measurements really to understand their history, the semantic content, the state of conservation (Basilissi et al., 2017; Kassahun Bekele et al., 2018). For the implementation of driven workflows, it is extremely important the deployment of ad-hoc technologies for reality-based 3D documentation by digital models. The creation and management of a digital surface reconstruction should be sustained in terms of processing requirements, geometric aspects, appearance attributes and overall digital projects manageability, as time-cost ration, interoperability constraints, heavyweight files dimensions and quality safeguard in compression methods, etc... It should be considered that the success of navigation and immersive aspects are sometimes as important as the ability to obtain high details all around. For maximizing the sustainability in the overall process related to these 3D models, new rapid and low-cost techniques are under observation in the applied research for their multi-purposes capability in responding to multi-scale and multi-content needs.

The framework, in which the presented research is a part of, is the experimenting on skills and critical points of a multi-sensor and multi-scale project of extended metric documentation about a segment of the urban landscape Heritage in Turin. The goal of the followed approach is connected to the "Torino 1911" project, led by the University of California San Diego (UCSD) in cooperation with the Politecnico di Torino: it is conceived for a virtual and immersive visualization of the Turin 1911 International "Fabulous Exposition" event, settled in the Valentino Park (Della Coletta, 2012). The idea is to use the acquired digital data of particular heritage structures by students, researchers and other users for explore the site's buildings and artefacts, ultimately recreating an interactive, virtual-reality experience. In particular, the paper will focus on a test belonging to an ongoing research about the potentialities of the descriptive capabilities of a handheld Mobile Mapping System (MMS) scanner based on Simultaneous Localization and Mapping (SLAM) technology: the ZEB REVO Real Time (Cadge, 2016) by GeoSLAM (2017 release) (https://geoslam.com). Different issues are presented hereafter related to two articulated and multilevel spaces as test studies: the Valentino Castle and the "Borgo Medievale" (Par.2). The paper faced with the ZEB point cloud validation and the difference between LiDAR approach and the ZEB Revo results connected to the drift error are evaluated as well (Par.3). Finally, an analysis about the possibility of colouring the ZEB point clouds using different proposed methods is reported (Par.4). 


\subsection{D models enhancing navigation purposes in the "Torino 1911" project}

In the Torino 1911 exposition, celebrateing the industrial fervor in the west part of Italy with its robust and explosive production of "goods, science and technology, national boundary and Capital" (Best \& Kellner, 1991), the architects created a fantastic city (Figure 1) on both banks of the Po River. The decoration was done with superfluous capitals as no one had ever seen before, with fountains, waterfalls and tapis roulant, fastigiums, porticos, statues of Victories with rustling veils, eagles with outstretched wings, angels playing trumpets (Della Coletta, 2012). The Torino 1911 project needs to facilitate constructive encounters between technologies: the old world's fair and new digital ones. The objective could be followed with ad-hoc realized tools that allow users to investigate exposition artifacts (in this first step, the area of the Valentino park). The idea is to allow users to move freely (changing the traditional computer animation approach with movements along fixed paths) in the reconstructed/surveyed scenario for exploring with virtual/digital reality equipment the area of the Torino 1911 exposition, as an integrated virtual word where wonder and critical interpretation, leisure and intellectual work involve and estrange each other in order to create new knowledge.

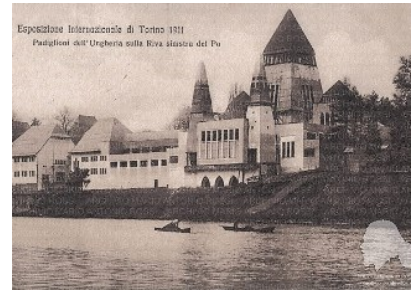

(a)

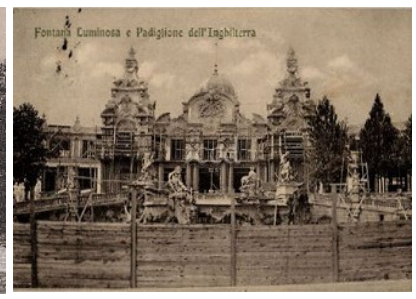

(b)
Figure 1. Hungary pavilion (a), UK pavilion (b) (http://www.italyworldsfairs.org, Della Coletta, 2012)

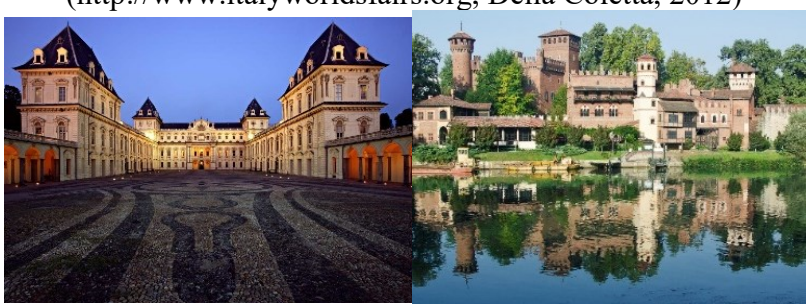

(a)

(b)

Figure 2. (a) A general view of the Valentino Castle (www.castellodelvalentino.polito.it). (b) A general view of the "Rocca Medioevale" (Image by Farshd Bazmandegan, UCSD).

For the first reconstruction step, the survey operations finalized to the 3D modelling of existing structures were carried out referring to the main floor of the Valentino Castle and the apartments of the "Borgo Medievale": both them, placed in the Valentino Park, have been involved in the 1911 World's Fair exposition. These structures have enclosed richly characterized volumes, with different featuring geometries, and precious decorative apparatus and require ah-hoc integrated approaches. The Castle (Figure 2, a) was one of the Savoy's royal family hunting residence. In the first floor, the honor floor decorated by important stucco and frescoes there were the residential rooms with two symmetrical apartments, originally built by Cristina of France and Vittorio Amedeo of Savoy in XVII century: the "Salone d'Onore" (Great Salon), focus of following analysis, is in the center of a composition (Figure 3). After some subsequent rearrangements, the Castel is hosting the Department of Architecture and Design of the Politecnico di Torino. In the "Borgo Medievale" (Figure 2, b), built along the Po river and surrounded by walls, the Rocca and the village as a small cluster of houses and workshops, are a reconstruction of an ideal medieval hamlet composed by different architectures, based on real existing building spread in Piemonte region. The idea of the 1911 exposition committee was to offer an artistic and evocative example of the gothic style. They were hosting the Ancient Art section during the 1884 International Exhibition, representing an example of the Italian style. Currently, the area with some tourists' attractions can be visited together with a museum of itself, in the most representative Rocca.

The Caste and the Village are thus two architectural ensembles composed by several buildings, characterized by different levels and rooms. In particular, the present paper considers two main areas: the main floor of the Castle as an example of a geometrically composite indoor environment distributed on a single level floor, and the inner courtyard in the Rocca with the distribution system to the other level floors.

In these heritage types of scenarios, many consolidated directions for 3D data acquisition and management have been frequently proposed, for what it concerns the geomatics approaching to an extensive documentation of articulated heritage complexes. The solutions based on multi-sensor image-based and range-based approaches, as close-range photogrammetry (CRP) and terrestrial laser scanning (TLS) systems, have been generally deployed in endorsed workflows, to overcome multi-scale and multiresolution issues in outdoor and indoor spaces for landscape and architectural reconstruction and analysis (Tucci, Bonora, Conti, \& Fiorini, 2017; Balletti, Guerra, Scocca, \& Gottardi, 2015; Guidi et al., 2009). As usual, it is well-know that they require time spending operations to survey and processing data referred to large areas. For these reasons, in some architectural large and/or compound environments, usually in case of uneasy access and articulated extents like the abovementioned ones, the consolidated surveying approaches often meet their applicability limits. They require time spending operations and concern especially on maneuverability matters and within the visualization-based optimization aims. Consequently, on one hand they derive from the extreme density of produced data unavoidably to be subsequently selected, reduced, simplified and optimized for reconstruction and navigation purposes.

Since the objective is to easily visualize the acquired information in order to improve the digital learning capability with 3D models displayed and navigable using virtual reality equipment, a first analysis on the possibility of the modern technologies and surveying methods was addressed as initial step of the project. The crucial issues are related to the accuracy control in these situations and the scale achievement in such kind of metric survey solutions according to the expected modelling issues.

\subsection{The solution of a portable Mobile Mapping System}

For these reasons, a more suitable technological solution based on a mobile sensor fusion approach seems to be the implementation of a Mobile Mapping Systems (MMSs) based on Simultaneous Localization and Mapping (SLAM) technology as solution of the positioning problem (Bellekens, Spruyt, \& Weyn, 2014; Riisgaard, 2005), now developing in various fields of application. It proves to be a flexible and competitive instrument to improve the time-consumption in data acquisition and files processing. In fact, the acquisition by MMSs in urban centres is quite largely develop in research experiences by means of vehicles and complex positioning systems based on ranging measurements, Global Navigation Satellite System (GNSS) and Inertial Measurement Unit (IMU) (Rodríguez-Gonzálvez, Jiménez Fernández-Palacios, Muñoz-Nieto, Arias-Sanchez, \& GonzalezAguilera, 2017; Toschi et al., 2017). However, a self-moving solution it is also useful in environmental survey for various 
purposes, especially in underground context or narrow spaces, like mines, caves and enclosed passageways (Mandelli, Fassi, Perfetti, \& Polari, 2017). Therefore, besides several types of gathering devices for MMSs platforms - as vehicles, trolley, backpacks (Nocerino, Menna, Remondino, Toschi, \& RodríguezGonzálvez, 2017; Tucci, Visintini, Bonora, \& Parisi, 2018) - a handheld portable solution under investigation (Sammartano \& Spanò, 2018; Sammartano, 2017) is proposed: the SLAM-based ZEB REVO-RT by GeoSLAM. In this research, several integrated tests performed with the aforementioned ZEB REVORT portable instrument are reported in order to accurately describe the pro and cons of the followed approach and the open issues founded using this emerging surveying technology.

\subsection{Multi-scale and multi-sensor approach in "Torino 1911"}

The survey operations realized in the two-test sites were carried out using a multi-scale and multi-sensor approach. First of all, as usual when the main objective is the realization of a correct and complete metric survey, in order to have a common reference system in the complete area a general GNSS network has been realized. Furthermore, since the built heritage were analysed in its indoor area as well, the GNSS survey has been integrated with some second order network measured with traditional Total Station equipment. Finally starting from the networks vertexes, the artificial markers placed on the surveyed structures were measured in order to use them in the related data processing parts (orientation) equally for SLAM-based data, photogrammetry or LiDAR. The accuracy of the measured points follows the typical precision of an architectural surveys that is connected to the representation scale: in this case 1:100 (graphic error $\pm 2 \mathrm{~cm}$ ). The results of the network adjustment firstly and the further side shot calculation fit the planned accuracy (for each measured points RMS less than $1.5 \mathrm{~cm}$ in all the components: X, Y, Z).

The honour floor of the Valentino Castle.

The first floor of the Castle with the Great Salon the integrated survey has been carried out with LiDAR system, photogrammetric survey and SLAM-based scanning acquisition by ZEB REVO RT, in order to have different kind of data useful to investigate effectiveness in the strategies of complete reconstruction workflows.

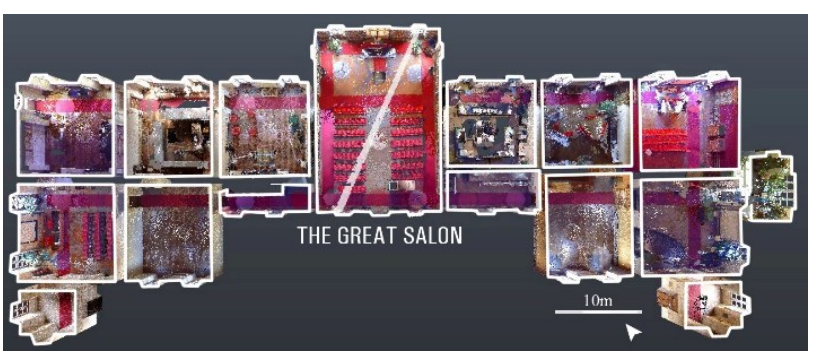

Figure 3. A plan of the complete LiDAR point cloud in the first floor of the Valentino Castle (the cloud is cut at $+2.5 \mathrm{~m}$ above the pavement). (Image by LabG4CH, Polito)

The acquired information furthermore was used for accuracy evaluation, comparison and naturally integration with the aim of using it in the virtual reconstruction framework. The LiDAR acquisition was performed using the Faro Focus ${ }^{3 \mathrm{D}}$ X330; this laser scanner allows to measure $\mathrm{n}^{\circ} 976.000$ points per second with a phase-shift technology ranging from $0.6 \mathrm{~m}$ to $330 \mathrm{~m}$, the laser is in class 1 and several sensors such us GPS, compass, altimeter ecc. According to the shape of the different rooms, a total of $n^{\circ} 82$ scans were acquired (almost $18 \mathrm{~h}$ acquisition time, for a documented surface of about $900 \mathrm{~m}^{2}$ ). The data processing has been carried out using the traditional approach performed in the scientific community and research group of the Politecnico di Torino
(Chiabrando et al., 2017; Gressin et al., 2013; Grussenmeyer et al., 2011, Muñumer \& Lerma 2015). The scans registration was based on ICP algorithm and target tie points. The accuracy control on the processing is: $1.35 \mathrm{~mm}$ mean value on ICP-like clouds fitting, and $3.2 \mathrm{~mm}$ mean value and $1.25 \mathrm{~mm}$ st.dev for RMSE on target tie points. In the following Figure 3 a cut section of the complete point clouds of the main floor reports the extension of the TLS project. The images acquisition process was realized testing different parallel approaches with increasing resolution sensors in the data collection. First, a complete photogrammetric acquisition was realized in each room, with large overlapping, for 3D modelling and high-resolution surfaces texturizing purposes. A DSLR camera, the Sony ILCE-7RM2 with $12 \mathrm{~mm}$ focal, was employed. In parallel, a low cost acquisition was performed using a steadycam, Osmo+ by DJI, (Calantropio, Patrucco, Sammartano, \& Teppati Losè, 2017) in order to test a more rapid and low cost acquisition system. For the colourizing phase proposed for the point cloud acquired by the SLAM-based ZEB scanner, the use of the photogrammetric data from steadycamera will be coupled and compared with those ones from the photogrammetric processing of the images from the ZebCam equipping the Zeb REVO RT system (GOPRO HERO4 session). The acquired data were then processed using Structure from Motion photogrammetric solution (Aicardi, Chiabrando, Lingua, \& Noardo, 2018; Chiabrando \& Spanò, 2013; Remondino, Spera, Nocerino, Menna, \& Nex, 2014; Westoby, Brasington, Glasser, Hambrey, \& Reynolds, 2012) in order to realize a complete photogrammetric model, to be integrated with the other acquisitions as well. In the framework of rapid mapping approach, several acquisitions using the ZEB REVO RT - with a tablet for real time view Figure 4 - were realized with an average duration of 10minutes. Different trajectories plans are focused for deeply evaluate the system in the different surveyed scenario. An accurate description of all the proposed workflow is reported (Par.3).
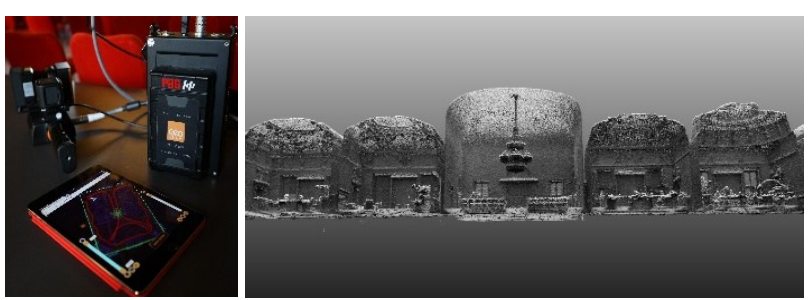

Figure 4. The ZEB REVO (left), 3D view of the scan acquired in the Valentino Castle (right). (Image by LabG4CH, Polito)

The apartments of the Medieval Rocca.

A complete survey of the "Borgo Medioevale", this complex indoor/outdoor scenario, is a very interesting challenge for more than one reason: tight spaces, large number of rooms, different levels and poor illumination (according to the typical medieval forma mentis).

The well consolidated techniques encountered several problems in this kind of scenario. First of all, the LiDAR approach was used for accurately documents all the rooms, by means of the same scanner described before (Faro Focus 3D X330). In order to describe the ground and first floor (Figure 5, left) of the Rocca, $\mathrm{n}^{\circ} 93$ scans were needed to be acquired and it was very time spending (almost $22 \mathrm{~h}$ acquisition time, for a documented surface of about $1000 \mathrm{~m}^{2}$ ). The scans registration was based on ICP algorithm and target tie points. The accuracy control on the processing is: $1.05 \mathrm{~mm}$ mean value on ICP-like clouds fitting, and $4.65 \mathrm{~mm}$ mean value and $2.69 \mathrm{~mm}$ st.dev for RMSE on target tie points. Moreover, the photogrammetric survey was performed as well: the poor illumination of the rooms required again a large amount of time in order to artificially illuminate the area. Therefore, only some rooms and decorated ceilings were imaged using a photogrammetric approach. In parallel, to consolidated 
high resolution DSLR cameras for image acquisition, low cost devices have been tested too.

According to the main objective of the survey, in the Rocca the ZEB REVO RT was employed following an articulated system of trajectories covering the compound system of the rooms' apartment (Figure 5, right). Certainly, it is exactly in this kind of building environment where the use of SLAM system shows the great advantage of integrating fast acquisition with accurate geometric reconstruction. The results and performed tests using the ZEB REVO are reported in the next sections.
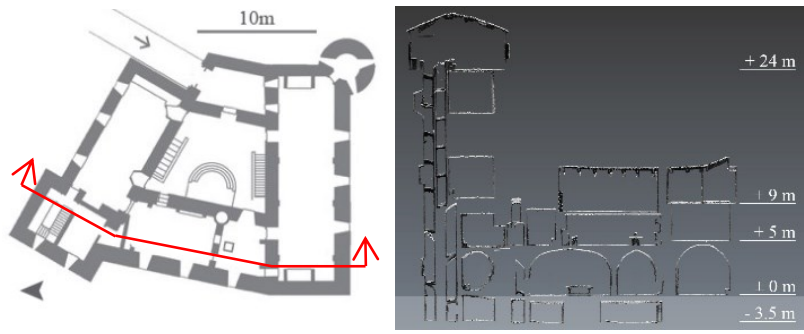

Figure 5. Section of the complexity in the vertical articulation of the Rocca apartments, from multiple ZEB scanning data (right). Schema of the ground floor of the Rocca, in red line, the vertical section (left).

\section{MMS POINT CLOUDS AND 3D GEOMETRIC RECONSTRUCTION}

The proposed investigation on this SLAM-based system for rapid mapping tries to deepen, aside from the specific geometric detail accuracy, the aspects related to the trajectories execution in relation to the $3 \mathrm{D}$ reconstruction performances in complex environments. A single-level and a multi-level mapping are thus proposed, considering different variables both in the mapping execution and in the data post-processing phases, in order to evaluate predictable drift errors that may affect the global accuracy in volumes reconstruction.

In fact, the mapping system operation is based on SLAM algorithm solving both for incremental motion estimation and global point cloud registration: a crucial relation exists between the travelled trajectory $[\mathrm{T}]$ and the geometric features of the surveyed object. The LiDAR profiles $[\mathrm{p}]$ are aligned each other progressively, based on the Iterative Closest Point (ICP) algorithm, during the mapping phase, according to the recognition of featuring geometric patterns extracted: their quality is fundamental also to align subsequent segments of the ZEB mapping profiles. Then, a global optimization is performed by the system, with the purpose of minimizing along the $T(\tau)$, following the RMSE principle, the errors between $p(\tau)$ on the surfaces matching and deviations indicated by the measured IMU accelerations and rotational velocities defining possible degrees-of-freedom (DOF) of $T(\tau)$ (Sammartano \& Spanò, 2018). Some variables should be thus took into consideration in the validation strategy, especially in such kind of wide setting with complex volumes and lengthy mapping. The influence of speed and type of $T(\tau)$; the preference of geometrically rich environments (no too extensive regular and no smooth surfaces); the presence of objects/people as well, increasing the noise effects of $p(\tau)$ registration; the retracing trajectory lines in already travelled segments using outward and return mode, helping the SLAM algorithm to have more rigidity in the $3 \mathrm{D}$ reconstruction; performing closed loops in the same position of the starting point to facilitate the SLAM algorithm and the IMU data to weight transition and rotation and to perform the best alignment minimizing errors along the trajectory, minimizing drift errors. The ZEB REVO-RT last release allows monitoring this process during the acquisition via mobile device connected by WI-FI signal to the ZEB data logger (Figure 4): it transmits in real time the $p(\tau)$ and the travelled $T(\tau)$ showing a preview of the mapping performance and the $T(\tau)$. The presence of the WI-FI to solve the data transmission has been marked as a critical variable affecting the real-time alignment system, both in the $p(\tau)$ registration and in the ZEBcam video synchronization. For example, in Figure 6 left, a registration error of about more than $1 \mathrm{~m}$ is visible on the tablet in real time, after the end of acquisition performed in an articulated and multi-level scan inside an enclosed circular tower of the Rocca: the arrival point is shifted from the start, as well as the starting room. In the preliminary optimization processing developed by the instrument at the end of each acquisition, the SLAM-based algorithm tries to correct the errors delivering a first $3 \mathrm{D}$ point cloud to be analyzed and, if necessary, maybe reprocessed by the GeoSLAM software.

In this case the optimization failed. Instead, the merging process - performed by the software, Figure 6 right, operated with another scan including this segment, partially adjusted the trajectory, realigning the two rooms but failed in the tower volume that results duplicated and affected by noise effect. The system may require different possible processing modes: apart from the first optimized point cloud performed by instrument, more focused reprocessing solutions are available in the GeoSLAM Hub platform.
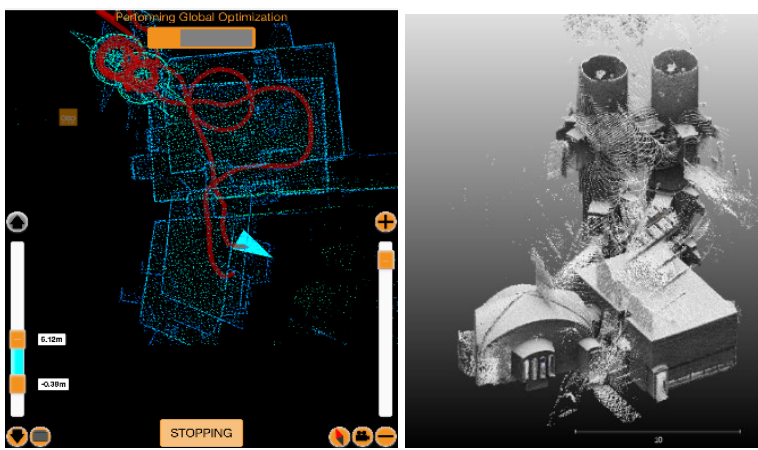

Figure 6. SLAM-base registration errors. (left) The real-time result in a multi-level scan inside the tower. (right) The result of the merge processing with another scan.

The processing parameters concern the possibility to improve the SLAM-based alignment of the first raw 3D data with a process reexecution, and they are going to operate locally or globally to adjust the $T(\tau)$ in the $p(\tau)$ registration. In the test, both standard recommended parameters and personalized parameters have been tested. A different processing solution, available for multiple scans data covering common trajectory segments, is the merge function algorithm, that can be defined as an alignment of different ZEB scan data based on a non-rigid registration, i.e. the trajectories and thus the 3D data are deformed and not only roto-translated. For example, in the merge processing, Figure 7, starting from two misaligned and partially matching scans (left), after a manual alignment based on roto-translation (right) operates a re-computation of trajectories based on both scan dataset, improving the results (see next paragraph).

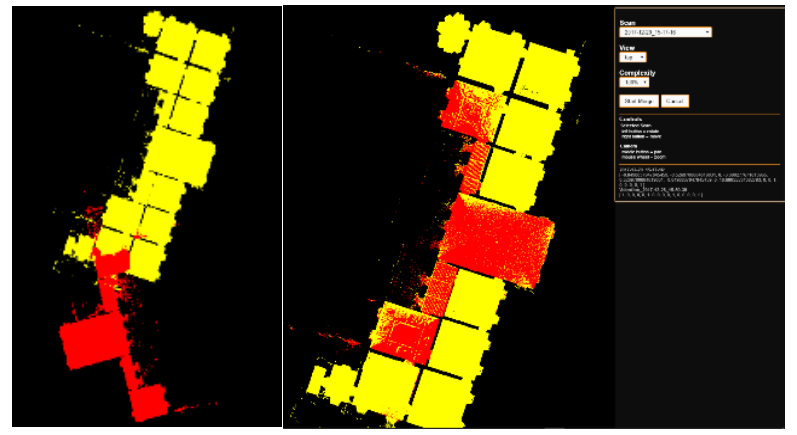

Figure 7. The merge process function in the GeoSLAM Hub SW 


\subsection{SLAM trajectory optimisation strategies: single-level floor}

In case of the main floor of the Castle, two trajectories were arranged and tested, aiming to evaluate and validate the best approach for such large-range mapping in quite complex volumes and geometrically characterized objects (smooth walls but lot of furnishings, and decorative apparatus, see Figure 4). The first $T(\tau)$ (Figure 8 , blue line, almost $6 \mathrm{~min}$ along $110 \mathrm{~m}, 0.3 \mathrm{~m} / \mathrm{s}$ speed), is a shorter and simpler one, performed with best practices execution a roundtrip -, travelling trajectory segments at least. The seconda non-roundtrip, but a close loop - (Figure 8, red line, almost 12min along $350 \mathrm{~m}, 0.5 \mathrm{~m} / \mathrm{s}$ speed), more complex, and complete across all the rooms on the floor coverage.

The reported analysis concerns the second trajectory (red). The processing solutions tested for the point cloud have been:

A. optimized by instrument: the first preliminary data

B. reprocessed by GeoSLAM SW- standard suggested parameters

C. reprocessed by GeoSLAM software according to personalized parameters: enhancing loop closing, using planar surfaces recognition and geometric features detection to help SLAM algorithm in the local processing, and increasing convergence threshold (i.e. the number of iteration in the SLAM progressive alignment optimization), in the global processing.

D. subjected to the merge process with the first scan (blue) An important aspect that is not secondary at all is considering how to perform the assessment, i.e. how to evaluate the presence of errors and their distribution according to reference surface (here TLS). In this sense, different levels of rigidity are tested. The resulting assessment are proposed, as well, as a possible optimal registration strategy in case of extended mapping projects.

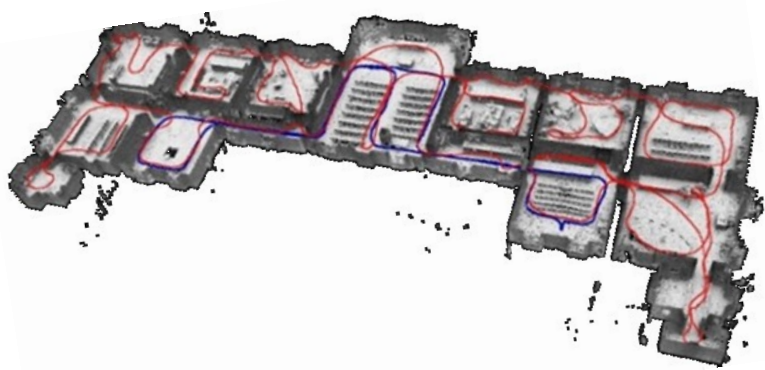

Figure 8. The two trajectories followed in the main floor of the Valentino Castle during the ZEB acquisitions

This is planned in order to minimizing the use of dense TLS point clouds scanning data as much as necessary and privilege the use of MMS wherever this level of detail is needed, as in this case of $3 \mathrm{D}$ modelling aimed at navigation purposes.

For example, in the Great Salon the density values are 77 (points $/ \mathrm{m}^{2}$ ) for TLS LiDAR against 25 (points $/ \mathrm{m}^{2}$ ) collected by the ZEB REVO system, with a global 3:1 ration between TLS and ZEB (Table 4.introduced further on).

The alignment modes, organized in different rigidity principles, for the mapping drift errors evaluation and a global overall accuracy assessment in the reconstruction, are based on ICP-like algorithm to operate a point cloud best-fitting with TLS ground-truth:

I. Global alignment, on the whole floor

II. Single local alignment, fixing the start room

III. Double local alignment, fixed at opposites

First of all, the config.I in the point cloud alignment is surely the one in which the error is distributed and thus appears lower $(\sim 8.5 \mathrm{~cm})$ with high st.dev. To evaluate the actual drift error, with Config.II, the alignment is operated in the starting room as fix point (Figure 9, plan, black circle), and the control measurements are extracted in the opposite corner room (Figure 9, Figure 10, “O” sample) according to Euclidean distances, split in $\mathrm{X}, \mathrm{Y}, \mathrm{Z}$ vectors.

\begin{tabular}{|c|c|c|c|c|c|c|c|c|}
\hline \multirow{3}{*}{\multicolumn{2}{|c|}{$(\mathrm{cm})$}} & \multirow{2}{*}{\multicolumn{2}{|c|}{$\begin{array}{l}\text { I - Global } \\
\text { alignment }\end{array}$}} & \multicolumn{5}{|c|}{ II - Single local alignment } \\
\hline & & & & \multicolumn{2}{|c|}{$\begin{array}{c}\text { ICP on } \\
\text { starting room }\end{array}$} & \multicolumn{3}{|c|}{$\begin{array}{c}\text { Max error at } \\
\text { opposite corner }\end{array}$} \\
\hline & & Mean & St.dev & Mean & St.dev & $\mathrm{X}$ & Y & Z \\
\hline \multirow{4}{*}{ 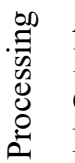 } & & 9.3 & 16.5 & 3.9 & 5.0 & 8.8 & 21.9 & 19.8 \\
\hline & B & 8.6 & 14.3 & 2.5 & 3.3 & 8.6 & 30.2 & 26.4 \\
\hline & C & 8.4 & 10.2 & 2.7 & 3.7 & 8.6 & 40.9 & 14.8 \\
\hline & D & 8.1 & 14.2 & 2.9 & 3.8 & 5.8 & 13.6 & 17.4 \\
\hline
\end{tabular}

Table 1. Alignment configuration I-II according to A, B, C, D processing

In (Table 1) are reported the ICP values both on the single room, indicating the good performance of the SLAM-based in the local reconstruction, and at the maximum vector error, as in Figure 10 (variable in $\mathrm{Z}$; around $8 \mathrm{~cm}$ in $\mathrm{X}$, demonstrating a non-actual dilatation of $3 \mathrm{D}$ reconstruction, and up to $40 \mathrm{~cm}$ along $\mathrm{Y}$ direction): the ration between $\mathrm{X}$ and $\mathrm{Y}$ directions are proving the typical drift error affecting this type of trajectory.

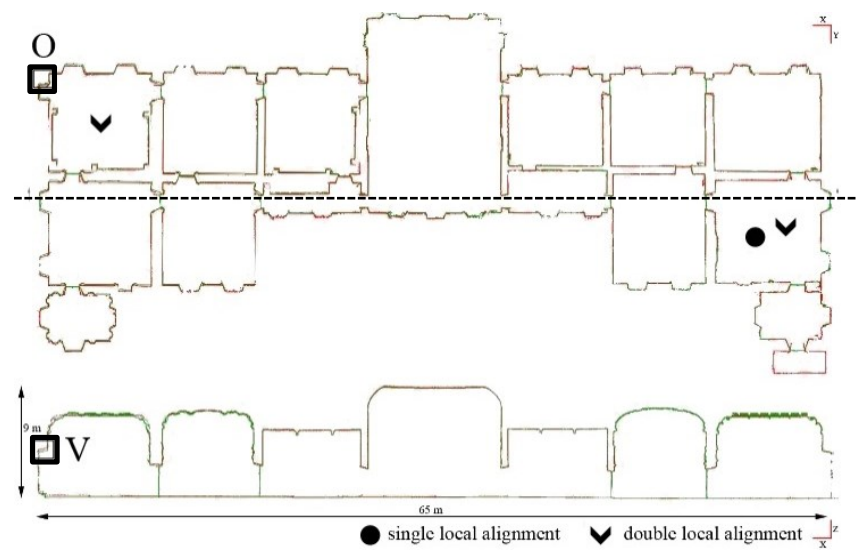

Figure 9. Planimetric representation (top) and vertical cut section (down), planimetrically positioned in dot line of the whole floor. Indication of the start/end point for the single local alignment (I) red, and the two fixing points for the double local alignment (III), blue.

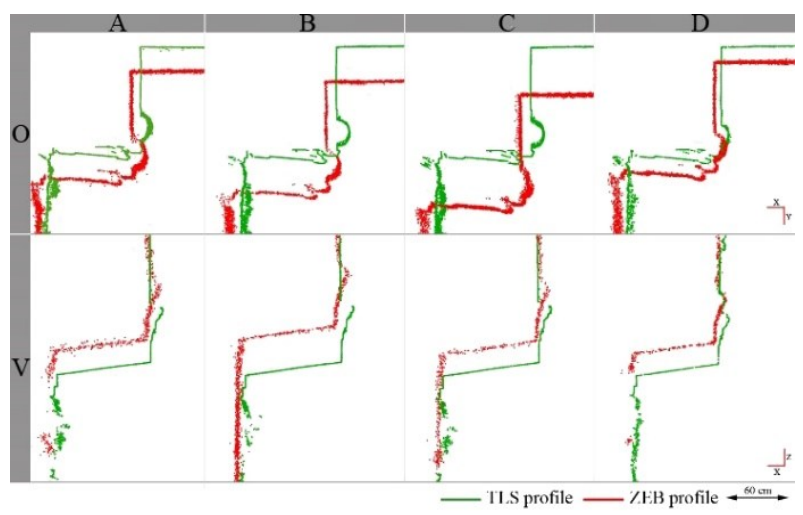

Figure 10. Configuration II, single local alignment. Misalignment errors detected in opposite corners in case of the single local point alignment, related to the $\mathrm{O}$ and $\mathrm{V}$ areas, pinpointed in Figure 9, according to the processing steps A, B, C, D: O in the horizontal slice, $\mathrm{V}$ in the vertical slice.

Then, Config.III will be considered to fix with more rigidity the system and thus evaluate more precisely the error drift. It is 
necessary to outline a general consideration about the actual efficacy of applying re-processing operation for the improvement of ZEB scans accuracy. It is possible to point out a real decreasing of noise and outliers' errors from the first optimization (A) to the optimization processing (B) and (C): (Table 1) st.dev values improved in parallel to a non-significant upgrading of mean value. Instead, the (D) processing operated mainly regarding the global correction of the drift error, as visible in Table 1 last line, and Figure 10 (D). The config.III is based on a more rigid alignment to evaluate efficiency of using only two reference objects to manage ZEB scans, as in Figure 9 (arrows). Statistical values are reported in Table 2. They show quite improved values, mainly with (D) process, and thus validate the possibility to operatively perform multiple and partially overlapping scans in articulated settings and solve the relative alignment solution with the merge process, and the global alignment with the use of even just few (here, two at extreme sides) reference surfaces (as georeferenced TLS scans).

\begin{tabular}{|c|c|c|c|c|c|c|}
\hline \multirow{2}{*}{\multicolumn{2}{|c|}{ III }} & \multicolumn{5}{|c|}{ Double local alignment } \\
\hline & & \multicolumn{2}{|c|}{$\begin{array}{l}\text { Local ICP on } \\
2 \text { room }\end{array}$} & \multicolumn{3}{|c|}{$\begin{array}{c}\text { Max error at } \\
\text { opposite corners }\end{array}$} \\
\hline \multicolumn{2}{|c|}{$(\mathrm{cm})$} & Mean & St.dev & $X$ & $\mathrm{Y}$ & Z \\
\hline \multirow{4}{*}{ b } & & 9.8 & 13.1 & 1.1 & 1.1 & 1.0 \\
\hline & B & 7.8 & 11.5 & 0.1 & -4.9 & 11.5 \\
\hline & $\mathbf{C}$ & 6.3 & 8.8 & -16.6 & 5.5 & -5.9 \\
\hline & D & 7.7 & 11.9 & -2.4 & -0.6 & 1.8 \\
\hline
\end{tabular}

Table 2. Alignment configuration III according to A, B, C, D processings

\subsection{Trajectory for multi-level 3D mapping: critical issues}

In case of multi-level mapping performed with the handheld ZEB system, as in the case of the Rocca apartments (Figure 5 right), the adopted strategy refers to the evaluation of possible drift errors in $\mathrm{Z}$ dimension compared to the TLS scanning project.

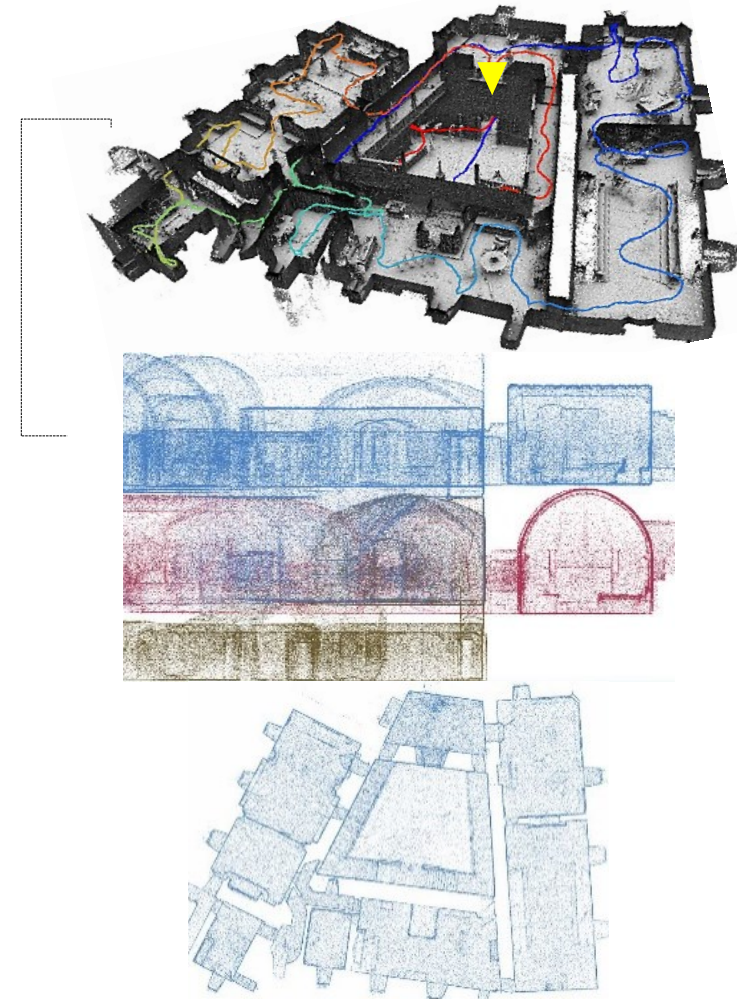

Figure 11. The side-view of the ZEB mapping along the three floors of the Rocca apartments (centre), and planar (down) and $3 \mathrm{D}$ view of the first floor (top), and its time-marked trajectory. Starting point at yellow arrow.
The considered trajectories have common starting from the ground floor, near the courtyard: it is important to focus that they belong to a barycentric reference surfaces compared to the trajectory progression around the balcony and rooms: this aspect is connected to a more rigid structure of the scanning project. The considered test-spaces are three (Figure 11 down): underground rooms, 8min scan (-3.5m height difference); ground floor courtyard and apartments, 9min scan; first floor apartment, 12 min scan $(+5 \mathrm{~m})$ (Figure 11 top).

The types of tested ZEB data are two (not-being reprocessed):

A. The preliminary optimized scans by instrument, singularly aligned to ground floor

B. The jointly merged scans

\begin{tabular}{|c|c|c|c|c|c|c|}
\hline \multirow{3}{*}{$(\mathrm{cm})$} & \multicolumn{3}{|c|}{ A. $1^{\text {st }}$ optimisation } & \multicolumn{3}{|c|}{ B. Merge processing } \\
\hline & \multicolumn{2}{|c|}{$\begin{array}{c}\text { Local ICP on } \\
\text { inner courtyard }\end{array}$} & \multirow{2}{*}{$\underset{\text { drift }}{Z}$} & \multicolumn{2}{|c|}{$\begin{array}{l}\text { Global ICP on the } \\
\text { floors }\end{array}$} & \multirow{2}{*}{$\begin{array}{c}\mathrm{Z} \\
\mathrm{drift}\end{array}$} \\
\hline & Mean & St.dev & & Mean & St.dev & \\
\hline Underground & 1.9 & 2.8 & 2.3 & & & 2.9 \\
\hline Ground floor & 2.3 & 3.4 & 2.2 & 3.1 & 5.3 & 2.4 \\
\hline First floor & 2.6 & 4.1 & 3.5 & & & 2.7 \\
\hline
\end{tabular}

Table 3. Statistical values of the accuracy assessment with TLS reference and evaluation of $\mathrm{Z}$ drift error on $\mathrm{ZEB}$ scans

The values reported in Table 3 firstly display the result of the point cloud preliminary optimization if each scan is only related to the starting point at ground floor: the assessment is pinpointed with the ICP average value on the inner courtyard, and in the $Z$ direction error (max $Z$ error in the $1^{\text {st }}$ floor, $3.5 \mathrm{~cm}$ ). The values of distributed errors are localized in limited ranges (between 1.9 and $4.1 \mathrm{~cm}$ ). Moreover, the really agreeable results figure out after the performing of the merge process (last columns) involving the joint trajectories of the three scans. It demonstrates the importance of the establishment of a rigidity in the structure of ZEB scans trajectories during the $3 \mathrm{D}$ mapping. The final average values defined for the global merged scans by MMS on the reference TLS surfaces are pinpointed as almost $3 \mathrm{~cm}$ value, with $5 \mathrm{~cm}$ of variability Table 3 .

\section{RADIOMETRIC ASEPCTS FEATURING THE ACHEIVABLE ZEB-BASED 3D MODELS}

Together with geometric aspects, the 3D reconstruction, performed by ZEB handheld MMS scanner for navigation purposes, should respond to effectiveness in appearance attributes too. Until the previous ZEB REVO release, the lack of radiometric data in the $3 \mathrm{D}$ points was a critical issue to be undertake. In fact, within the implementation of the portable scanning system by GeoSLAM, the use of the ZEBcam data (images/videos) are now available for multi-purposes investigations and possible interesting development. The wellknown GoPRO HERO session action cam is equipping the system since the ZEB REVO first release. The camera is now connected by cable to the data logger, and the relative position of the camera centre with the IMU internal to the rotating head is defined as calibrated known value. A direct alignment of video and trajectory is a possible solution proposed by the software implementation, and a more complex and experimental texturizing of the point cloud by ZEBcam image frames is proposed as added function. Both the solutions are based on a key-topic: the success in the registration and processing algorithm - that works with the trajectory and the IMU data - is strictly associated to the time variable. In this way, it is possible to associate the images frames from the video to the trajectory points positions. From that correlation, a direct navigation of the 3D scan via GeoSLAM Hub software platform is possible to preview the point cloud and the related images during the 
movements along the path. Once established the images position and orientation, the developed algorithm based on photogrammetric principles try to project radiometric data from images to the point cloud and proceed to the whole texturization of the cloud. This last procedure is still under investigation: the critical phase of the starting/stopping scan action - executed by the operator in coordination with the ZEBcam recording, but in separated moments - can often affect the success of the alignment results, so IMU data from trajectories frequently cannot be associated to the video data from the GeoSLAM Hub. For these reasons, at the moment the radiometric matter is faced up with proposed and compared alternatives image-based approaches. It will be systematized for the Great Salon of the Valentino Castle as sample case: here the appearance attributes of the surfaces are definitely more characterizing. The Great Salon is featured by following dimensions: (lxbxh) $17 \mathrm{~m} \times 11 \mathrm{~m} \times 9 \mathrm{~m}$, with almost $183 \mathrm{~m}^{2}$ surface and $1645 \mathrm{~m}^{3}$ volume. Here three photogrammetric approaches are tested and acquisitions are planned with adequate overlapping for overcome the problem of radiometric lack and develop the possibility to apply RGB data both to ZEB point cloud and derived ZEB mesh from photogrammetric process (Table 4):

A. the use of the video frames extracted by the customized ZEBcam. 30 frames/sec (extracted 1frame/sec) Figure 12, left;

B. the use of high resolution images by traditional DSLR camera acquisition. The SONY ILCE 7RM2, full-frame CMOS sensor 42MP (7952x5304px images), equipped with $12 \mathrm{~mm}$ focal lens;

C. the use of a steadycamera as proposal of an intermediate solution, Osmo + in video mode acquisition, with a 24 frames/sec video (extracted 1 frame/sec), Figure 12, left.

The images were processed with photogrammetric SfM approach in the well-known Agisoft Photoscan platform (www.agisoft.com). With the bundle block adjustment, the camera pose estimation is defined and the metric control on residual errors is reported in Table 4 . The dense point clouds will be considered and compared with the ones from LiDAR data (ZEB and TLS) as in Table 4.

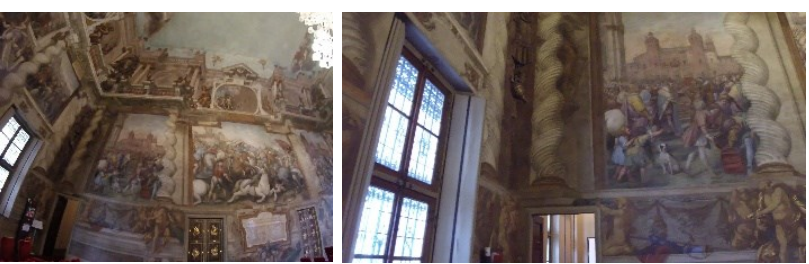

Figure 12. Comparison of video frames and FOV for the ZebCAM (left) and Osmo+ DJI (right) extracted for photogrammetric purposes.

\begin{tabular}{|c|c|c|c|c|}
\hline & & a-ZebCam & b - Sony & c - Osmo \\
\hline $\mathrm{n}^{\circ}$ images & & 238 & 202 & 473 \\
\hline Images file size & $M b$ & 0.6 & 44.2 & 1.6 \\
\hline Images resolutior & pixel & $1920 \times 1440$ & $5304 \times 7952$ & $4096 \times 2160$ \\
\hline $\mathrm{N}^{\circ} 18$ GCPs RMSE & $\mathrm{mm}$ & 13.56 & 5.71 & 9.06 \\
\hline $\mathrm{N}^{\circ} 6$ CPs RMSE & $\mathrm{mm}$ & 17.86 & 7.58 & 18.85 \\
\hline Acquisition time & $\min$ & 5 & $40 *$ & $10^{*}$ \\
\hline \multirow{3}{*}{$\begin{array}{c}\text { Density } \\
\text { (points } / m^{2} \text { ) }\end{array}$} & \multirow{3}{*}{$\begin{array}{l}\text { ZEB scan } \\
\text { TLS scan }\end{array}$} & 6,125 & 94,117 & 70,411 \\
\hline & & & 76,784 & \\
\hline & & & 25,651 & \\
\hline
\end{tabular}

Table 4. Photogrammetric datasets specifics. $(*)$ needs the added time value of ZEB mapping. Comparison with rangebased densities.

The image-based acquisitions are proposed as alternatives, as introduced, in order to test a rapid system for the radiometric data association to the 3D ZEB models for navigation purposes: the MMS point cloud is considered as well as the triangulated mesh surface generated from the MMS point cloud. In case of point cloud data, the radiometric values association is directly possible between two data: the ZEB points clouds Figure 13, and the photogrammetric one with RGB information. The OS Cloud Compare software (http://www.danielgm.net/cc/) allows the management of radiometric values between point clouds objects. In Figure 13, $a, b, c$ the results of the RGB data association by a visual comparison are visible, especially in the linked focuses where excerpts of each image from the different sensors radically differ for many aspects, i.e. image quality and resolution (Table 4). As expected, due to different densities between the point clouds, as well as image qualities, the RGB data is differently resampled and simplified to be assigned to the ZEB cloud. Moreover, considering instead the use of a meshed model in the navigation applications, a photogrammetric SfM project allows using the control of the camera pose for the texturizing process of a surface model. In fact, Photoscan software allows interoperability in data-import operation regarding mesh objects. For these reasons, the triangulated mesh with ZEB point cloud, computed and optimized as Figure 14 first image, has been imported in the photogrammetric projects containing oriented images. Then it was possible to compute the texture mapping as Figure 14, $a, b, c$, with different resolution qualities, as noticeable from a visual analysis.

In Table 5 some size variables are listed to be considered in the use of those textured mesh objects produced with rapid mapping SLAM-based handheld scanner. It is important to underline that acquisition times, together with files sizes, could influence very much the decision for the approach adoption. For example, in case of the ZEB REVO-RT integrated solution (scanning+ZEBcam) the scanning and images times acquisition are coincident, but the radiometric quality is lower. The use of video frames for photogrammetric purposes from a low-cost steadycamera (Figure 13, c and Figure 14, c) displays easymanageable data and high-quality coloured point cloud and mesh surface, in which the time-cost balancing for the visual impact of 3D metric data results very successful.

\begin{tabular}{llccc} 
& \multicolumn{1}{c}{$M b$} & ${ }^{*}$.obj+*.jpeg size & *.obj size & *.jpeg size \\
\hline & SLAM-based & 20.1 & 20.084 & no texture \\
a & ZebCam & 56.7 & 43.507 & 13.202 \\
b & Sony & 72.2 & 43.519 & 28.704 \\
c & Osmo & 66.2 & 43.501 & 22.676 \\
\hline
\end{tabular}

Table 5. ZEB-based triangulated surfaces files texturized according to $a, b, c$

\section{FIRST CONCLUSION AND FUTURE PERSPECTIVES}

The present research proposes the challenge to investigate the best practices to obtain a rapid and accurate mapping with a SLAM-based solution, integrated with other survey methods too. The geometric and radiometric aspects are both crucial aspects of this innovative rapid mapping technique and should be take into consideration in case of visual virtual reconstructions, i.e if 3D point clouds and/or surface models are finalized to digital projects of immersive and interactive navigations. The analyses are connected not only on the operative times and resources, but also metric and visual quality results in various indoor/outdoor settings. For geometric reconstruction is necessary to pay a lot of attention in the planning of the ZEB acquisition in order to minimize the drift error that, as reported, is a crucial aspect for a correct survey of extended areas. Anyway, it is possible to state that, for the objective of the work connected to navigation purpose, this kind of accuracy problems are not crucial compared to the ones that face with the radiometric contents of the point clouds and surface models. According to this last aspect is important to point out the problem derived using the connected GOPRO camera in terms of synchronization and image quality. 


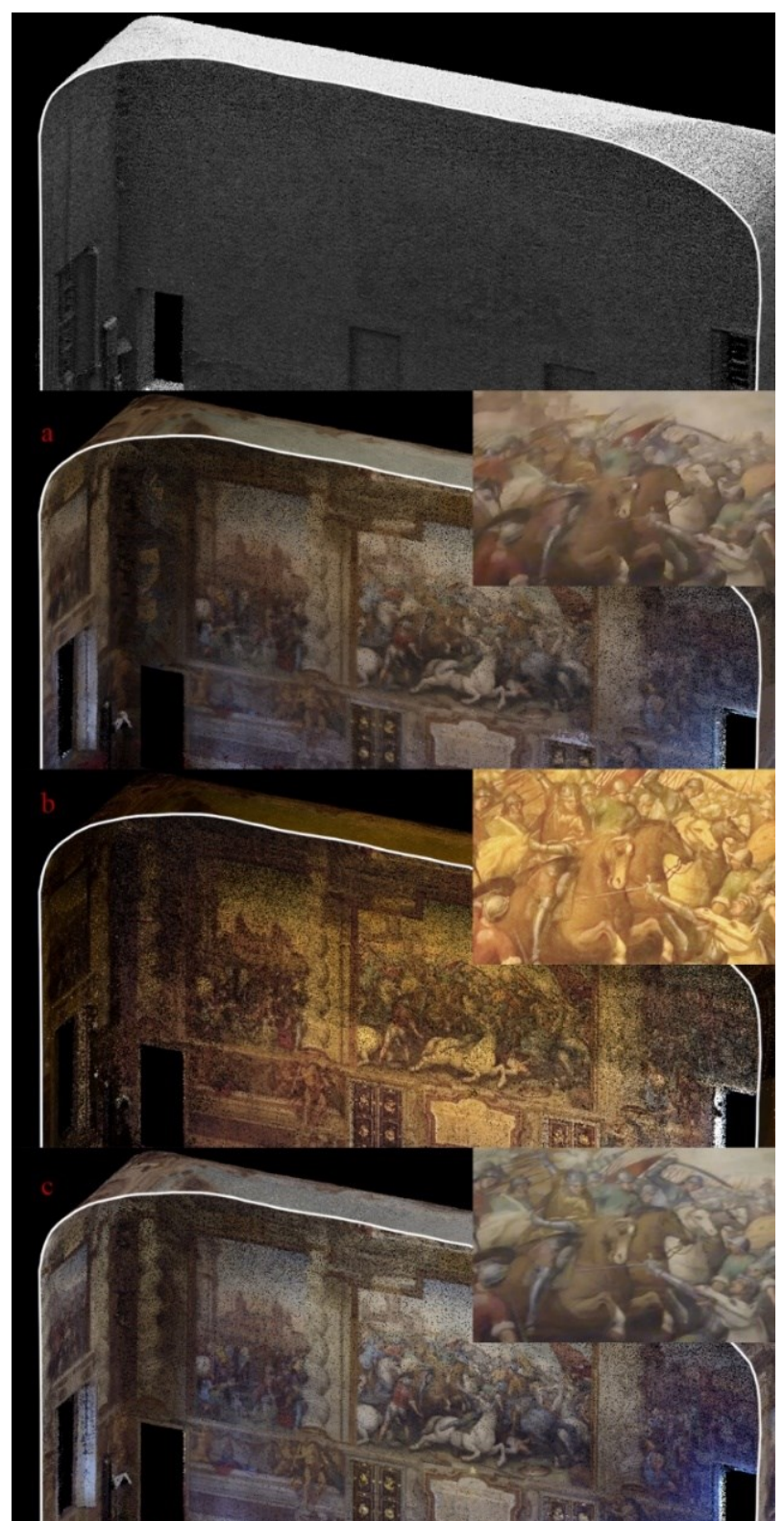

Figure 13. ZEB Point clouds with associated radiometric values: top ZEB point cloud in shaded view. Photogrammetric point clouds: $a$, ZEBcam; $b$, Sony ILCE; $c$, steadicamera. For each point cloud model, a zoomed view of the image used in the photogrammetric process.

The steadycam proposed solution allows to guarantee a fast acquisition with excellent results in terms of quality and accuracy of the final textured output, both in case of point cloud or textured mesh. Moreover, in terms of data management, low size files are more useful in on-line web visualization for real time rendering and navigation. For 3D navigable model is important to work in the direction of multi-scale modelling in order to optimize each part of the visualization that will increase the detail according to the scale of the visualization; the pyramidal approach followed by the gaming engine platforms should probably be the best way for documenting cultural heritage environment as well. Some further analysis and tests need to be performed in this scenario, but the attained results partially investigated in the present analysis are a valuable promise from this powerful 3D mapping solution. Furthermore, they confirm the use of handheld SLAM system as a possible approach in endorsed mapping workflow to produce an accurate and easy navigable $3 \mathrm{D}$ model for documentation projects regarding Built Heritage domain.

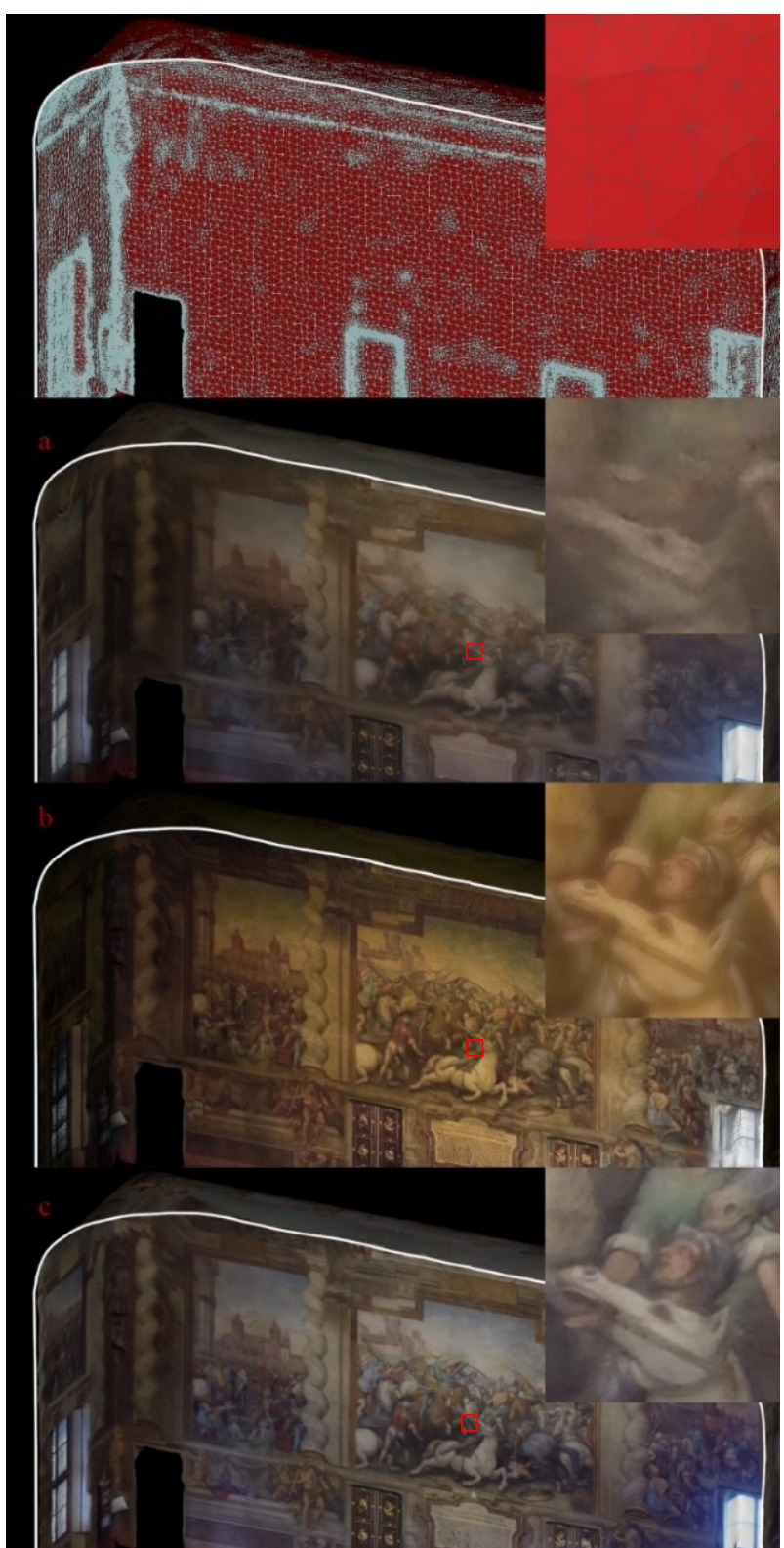

Figure 14. Triangulated surface generated by ZEB point clouds with associated photogrammetric texture. top, ZEB triangulated mesh in wireframe view. Texture from: $a$, ZEBcam frames; $b$, Sony ILCE images; $c$, steadicamera frames. For each textured mesh model, a zoomed view of about $50 \times 50 \mathrm{~cm}$.

\section{ACKNOWLEDGEMENTS}

The authors would like to express their sincere gratitude to the following colleagues from the Laboratory of Geomatics for Cultural Heritage $(\mathrm{G} 4 \mathrm{CH})$ of the Politecnico di TorinoDepartment of Architecture and Design: L. Teppati Losè, G. Patrucco and E. Colucci for data acquisition and processing. A special thanks to the researcher of UCS University of California San Diego for the cold acquisition campaign in the Borgo Medioevale and Valentino Castle of December 2017 D. Rissolo, E. Lo and F. Bazmandegan. Finally thank you to C. Bonfanti from MESA srl for ZEB Revo by GeoSLAM suggestions and helps.

\section{REFERENCES}

Aicardi, I., Chiabrando, F., Lingua, A. M., \& Noardo, F., 2018. Recent trends in cultural heritage 3D survey: The photogrammetric computer vision approach. Journal of Cultural 
Heritage. https://doi.org/10.1016/j.culher.2017.11.006

Basilissi, V., Bitelli, G., Callieri, M., Catalano, D., Dellepiane, M., Ponchio, F., ... Scopigno, R., 2017. Il restauro della Fontana del Nettuno a Bologna. Un sistema 3D web per la documentazione e la gestione dei dati. Archeomatica, (4), 30-35. http://ojs.mediageo.it/index.php/archeomatica/article/view/1398 $\% 3 \mathrm{E}$

Bellekens, B., Spruyt, V., \& Weyn, M., 2014. A Survey of Rigid 3D Pointcloud Registration Algorithms. AMBIENT 2014, The Fourth International Conference on Ambient Computing, Applications, Services and Technologies. 2014., (c), 8-13.

Best, S., Kellner, D., 1991. Post Modern Theory: Critical Interpretations. New York: Guilford print.

Cadge, S., 2016. Welcome to the ZEB REVOlution. GEOmedia, 22-26.

Calantropio, A., Patrucco, G., Sammartano, G., \& Teppati Losè, L., 2017. Low-cost sensors for rapid mapping of cultural heritage: first tests using a COTS Steadicamera. Applied Geomatics, 1-10. https://doi.org/10.1007/s12518-017-0199-6

Chiabrando, F., Spano, A. T., Sammartano, G., \& Teppati Lose, L., 2017. UAV oblique photogrammetry and lidar data acquisition for 3D documentation of the Hercules Fountain. Virtual Archaeology Review, 8(16), pp. 83-96.

Chiabrando, F., Donadio, E., \& Rinaudo, F., 2015. SfM for orthophoto generation: Awinning approach for cultural heritage knowledge. International Archives of the Photogrammetry, Remote Sensing and Spatial Information Sciences - ISPRS Archives, 40(5W7), https://doi.org/10.5194/isprsarchives-XL-5-W7-91-2015

Chiabrando, F., \& Spanò, A. T., 2013. Points clouds generation using tls and dense-matching techniques. A test on approachable accuracies of different tools. ISPRS Annals of Photogrammetry, Remote Sensing and Spatial Information Sciences, II-5/W1, 6772. https://doi.org/10.5194/isprsannals-II-5-W1-67-2013

Della Coletta, C., 2012. Of Work and Leisure: Digital World's Fairs and the Active Fairgoer. PMLA, Volume 127, Number 4, October 2012, pp. 939-946.

Evans, A., Romeo, M., Bahrehmand, A., Agenjo, J., \& Blat, J., 2014. 3D graphics on the web: A survey. https://doi.org/10.1016/j.cag.2014.02.002

Gressin, A., Mallet, C., Demantké, J., David, N., 2013. Towards 3D lidar point cloud registration improvement using optimal neighborhood knowledge. ISPRS journal of photogrammetry and remote sensing, 79 , pp. 240-251.

Grussenmeyer, P., Alby, E., Assali, P., Poitevin, V., Hullo, J. F., Smigiel, E., 2011. Accurate documentation in cultural heritage by merging TLS and high-resolution photogrammetric data. In Videometrics, Range Imaging, and Applications XI (Vol. 8085). International Society for Optics and Photonics.

Kassahun Bekele, M., Pierdicca, R., Frontoni, E., Malinverni, E. S., Gain, J., Bekele, M. K., ... Malinverni, E. S. (2018). A Survey of Augmented, Virtual, and Mixed Reality for Cultural Heritage. ACM J. Comput. Cult. Herit. ACM Journal on Computing and Cultural Heritage, 11(7). https://doi.org/10.1145/3145534

Lee, H., Lavoué, G., \& Dupont, F., 2012. Rate-distortion optimization for progressive compression of 3D mesh with color attributes. The Visual Computer, 28(2), 137-153. https://doi.org/10.1007/s00371-011-0602-y

Mandelli, A., Fassi, F., Perfetti, L., \& Polari, C., 2017. Testing different survey techniques to model architectonic narrow spaces. ISPRS - International Archives of the Photogrammetry, Remote Sensing and Spatial Information Sciences, XLII-2/W5, 505-511. https://doi.org/10.5194/isprs-archives-XLII-2-W5-

\section{5-2017}

Muñumer, E., Lerma, J. L., 2015. Fusion of 3D data from different image-based and range-based sources for efficient heritage recording. In Digital Heritage, 2015 (Vol. 1, pp. 83-86). IEEE

Nocerino, E., Menna, F., Remondino, F., Toschi, I., \& Rodríguez-Gonzálvez, P., 2017. Investigation of indoor and outdoor performance of two portable mobile mapping systems. In F. Remondino \& M. R. Shortis (Eds.), Videometrics, Range Imaging, and Applications XIV, edited, SPIE 2017 (p. 103320I). https://doi.org/10.1117/12.2270761

Potenziani, M., Callieri, M., Dellepiane, M., Corsini, M., Ponchio, F., \& Scopigno, R., 2015. 3DHOP: 3D Heritage Online Presenter. Computers \& Graphics, 52, 129-141. https://doi.org/10.1016/J.CAG.2015.07.001

Puente, I., González-Jorge, H., Martínez-Sánchez, J., \& Arias, P., 2013. Review of mobile mapping and surveying technologies. Measurement, 46(7), 2127-2145. https://doi.org/10.1016/j.measurement.2013.03.006

Remondino, F., Spera, M. G., Nocerino, E., Menna, F., \& Nex, F., 2014. State of the art in high density image matching. The Photogrammetric Record, 29(146), 144-166. https://doi.org/10.1111/phor.12063

Riisgaard, S., 2005. SLAM for Dummies: A tutorial approach to Simultaneous Localization and Mapping. Citeulike.org. https://doi.org/10.1017/S0025315400002526

Rodríguez-Gonzálvez, P., Jiménez Fernández-Palacios, B., Muñoz-Nieto, Á., Arias-Sanchez, P., \& Gonzalez-Aguilera, D., 2017. Mobile LiDAR System: New Possibilities for the Documentation and Dissemination of Large Cultural Heritage $\begin{array}{llll}\text { Sites. } & \text { Remote } & \text { Sensing, } & 9(3),\end{array}$ https://doi.org/10.3390/rs9030189

Sammartano G, Spanò A., 2018. Point clouds by SLAM-based mobile mapping systems: Accuracy and geometric contents validation in multi-sensor survey and stand-alone acquisition. Accepted in: Applied Geomatics, Special Issue (forthcoming)

Sammartano, G., 2017. Optimization of 3D multi-sensor models for damage assessment in emergency context: first tests on rapid mapping in the 2016 Italian earthquake. In F. Remondino, A. Georgopoulos, D. Gonzalez-Aguilera, \& P. Agrafiotis (Eds.), Latest Developments in Reality-Based $3 D$ Surveying and Modeling. MDPI.

Spanò, A., Chiabrando, F., Dezzani, L., \& Prencipe, A., 2016. Digital Segusio: from models generation to urban reconstruction. Virtual Archaeology Review, 7(15), 87. https://doi.org/10.4995/var.2016.5874

Toschi, I., Ramos, M. M., Nocerino, E., Menna, F., Remondino, F., Moe, K., ... Fassi, F., 2017. Oblique photogrammetry supporting $3 \mathrm{~d}$ urban reconstruction of complex scenarios. ISPRS - International Archives of the Photogrammetry, Remote Sensing and Spatial Information Sciences, XLII-1/W1, 519-526. https://doi.org/10.5194/isprs-archives-XLII-1-W1-519-2017

Toschi, I., Rodríguez-Gonzálvez, P., Remondino, F., Minto, S., Orlandini, S., \& Fuller, A., 2015. Accuracy evaluation of a mobile mapping system with advanced statistical methods. ISPRS - International Archives of the Photogrammetry, Remote Sensing and Spatial Information Sciences, XL-5/W4(5W4), 245253. https://doi.org/10.5194/isprsarchives-XL-5-W4-245-2015

Tucci, G., Bonora, V., Conti, A., \& Fiorini, L., 2017. Digital Workflow for the Acquisition and Elaboration of 3D Data in a Monumental Complex: the Fortress of Saint John the Baptist in Florence. ISPRS - International Archives of the Photogrammetry, Remote Sensing and Spatial Information Sciences, XLII- 
2/W5(September), 679-686. https://doi.org/10.5194/isprsarchives-XLII-2-W5-679-2017

Tucci, G., Visintini, D., Bonora, V., \& Parisi, E., 2018. Examination of Indoor Mobile Mapping Systems in a Diversified Internal/External Test Field. Applied Sciences, 8(3), 401. https://doi.org/10.3390/app8030401

Westoby, M. J., Brasington, J., Glasser, N. F., Hambrey, M. J., \& Reynolds, J. M., 2012. "Structure-from-Motion" photogrammetry: A low-cost, effective tool for geoscience applications. Geomorphology, 179, 300-314. https://doi.org/10.1016/j.geomorph.2012.08.021 